tertiary referral centres have reported short-term outcomes and adverse events reported in the hospital setting only.

Methods To review the short and long-term efficacy and adverse events of IFX rescue therapy in patients with ASUC in a secondary care setting.

The authors rigorously reviewed hospital notes and primary care records of all patients treated with IFX as rescue therapy for steroid refractory ASUC in Exeter. The primary end point was colectomy-free survival. Secondary outcomes included physician and patient assessment of response and adverse events.

Results 32 patients received IFX for ASUC between December 2005 and November 2010, with a median follow-up postinfusion of 15 months (range $0-53$ IOR 33). On admission 30/32 patients met the Truelove and Witts criteria for ASUC. 2 patients had co-existing Clostridium difficile infection. Mean CRP at admission and prior to IFX was 48 and $28 \mathrm{mg} / \mathrm{l}$ respectively. The median time from admission to first dose of infliximab was 7 days (range $1-31$ IOR 7 ). $32 \%$ of patients received IFX within 1 month of diagnosis. One patient who came to colectomy was found to have ischaemic colitis with superimposed fulminant CMV colitis, rather than UC. Of the remaining 31 patients, 20/31 (65\%) received a 3 dose induction regimen. After the index admission $19 \%$ had maintenance therapy and $15 \%$ had episodic dosing.

$5(16 \%)$ had a colectomy at index admission. Global physician assessment and patient assessment deemed 8/31 (26\%) and 7/31 $(23 \%)$ as primary non-responders to IFX respectively. At 12 , 24 and 48 months follow-up 11 (48\%), 13 (55\%) and 14 (64\%) had undergone colectomy. Logistic regression analysis failed to identify any clinical or laboratory parameter that predicted outcome in this IFX treated group. Adverse events on index admission included one fulminate CMV colitis, one post-op wound infection and 2 mild infusion reactions. Adverse events on follow-up included 1 episode of $C$ difficile infection and 1 Campylobacter infection resulting in colectomy.

Conclusion This is the largest reported UK cohort of patients treated with IFX for ASUC, with the longest median follow-up. Response rates are comparable with previous studies, with $16 \%$ undergoing colectomy at index admission and $64 \%$ at 48 months. Comprehensive review of both primary and secondary care notes demonstrated an acceptable rate of adverse events with $13 \%$ developing serious infections, 2 at index admission and 2 while receiving IFX maintenance therapy.

Competing interests None.

Keywords infliximab, ulcerative colitis.

\section{PTH-053 INFLIXIMAB RESCUE THERAPY FOR STEROID REFRACTORY ACUTE SEVERE ULCERATIVE COLITIS IN THE EXETER IBD COHORT}

doi:10.1136/gut.2011.239301.454

0 Waters, ${ }^{1}$ M Saunders, 1,* M Clarke, ${ }^{1}$ T Daneshmend, ${ }^{1}$ T Ahmad ${ }^{1}$ Royal Devon and Exeter Hospital, Exeter, UK

Introduction Infliximab (IFX) has been shown to be efficacious in the treatment of steroid refractory acute severe ulcerative colitis (ASUC) with reduced rates of colectomy at index admission and at 3-year follow-up. Previous studies from 